\title{
Which heart rate variability index is an independent predictor of mortality in cirrhosis
}

Amar S. Bhogal ${ }^{1}$, Michele De Rui ${ }^{2}$, Donatella Pavanello², Ilias El-Azizi ${ }^{1}$, Sadia Rowshan ${ }^{1}$, Piero Amodio $^{2}$, Sara Montagnese ${ }^{2 *}$, Ali R Mani ${ }^{1 *}$

${ }^{1}$ Division of Medicine, University College London, London, UK

2 Department of Medicine, University of Padova, Padova, Italy

*Corresponding Authors:

Emails: a.r.mani@ucl.ac.uk, sara.montagnese@unipd.it

Tel/Fax: $+44(0) 2074332878$

Electronic Word Count: 3495

Financial Support:

Ali R Mani was supported by a Visiting Professor 2016 award (University of Padova Fondazione Cariparo).

List of Abbreviations:

CRP: C-Reactive Protein

CSDNN: Corrected Standard Deviation of inter-beat intervals

DFA: Detrended Fluctuation Analysis

HF: High Frequency

HRC: Heart Rate Characteristics

HRV: Heart Rate Variability

IL-6: Interleukin-6

LF: Low Frequency

MELD: Model for End-Stage Liver Disease

TNF- $\alpha$ : Tumour Necrosis Factor- $\alpha$

SampEn: Sample Entropy

SDNN: Standard Deviation of inter-beat intervals

VLF: Very Low Frequency 


\begin{abstract}
:
Background: Liver cirrhosis is associated with reduced heart rate variability (HRV), which indicates impaired integrity of cardiovascular control in this patient population. There are several different indices for HRV quantification. The present study was designed to: 1) determine which of the HRV indices is best at predicting mortality in patients with cirrhosis; 2) verify if such ability to predict mortality is independent of the severity of hepatic failure.

Methods: Ten minutes electrocardiogram was recorded in 74 patients with cirrhosis. Heart rate fluctuations were quantified using statistical, geometrical and non-linear analysis. The patients were followed-up for 18 months and information was collected on the occurrence of death/liver transplantation.
\end{abstract}

Results: During the follow-up period, 24 patients (32\%) died or were transplanted for hepatic decompensation. Cox's regression analysis showed that SDNN (total HRV), CSDNN (corrected SDNN), SD1 (short-term HRV), SD2 (long-terms HRV) and spectral indices could predict survival in these patients. However, only SD2 and CSDNN were shown to be independent of MELD in predicting survival. The prognostic value of HRV indices was independent of age, gender, use of beta blockers, and the aetiology of liver disease.

Conclusion: Two HRV indices were identified that could predict mortality in patients with cirrhosis, independently of MELD. These indices are potentially useful tools for survival prediction.

Key words: Cirrhosis, Heart rate variability, MELD, Survival 


\section{INTRODUCTION}

Liver cirrhosis is a global health burden worldwide causing around 1 million deaths annually with numbers rising (1). Cirrhosis is a disease with multisystem involvement (cardiovascular, renal, neurological, and immunological) and as such cirrhosis can lead to death through infection, hepatorenal syndrome, cardiovascular disease, gastrointestinal bleeding, and multiorgan failure (2). Median survival time for these patients is just above 78 months with compensated disease and much shorter at 29.5 months for decompensated disease (3). Therefore, predicting mortality in this population may offer a better way of stratifying -for purposes of treatment options and care optimisation. Current clinical indices used in prediction of mortality in cirrhosis (e.g. MELD) are based on measurement of biochemical markers. Changes in these biomarkers reflect hepatocellular dysfunction or some aspects of systemic complications such as hepatorenal syndrome. However, the multisystem involvement of cirrhosis is not reflected in these biochemical markers. Physiological markers (physiomarkers) exists that may enhance the prognostic value of MELD. For example, the addition of EEG analysis improves the prognostic value of MELD in cirrhotic patients awaiting transplantation (4). Heart Rate Variability (HRV) analysis is another physiomarker that has been used to assess the integrity of cardiovascular control system. Lower HRV in the general population is a predictor of mortality and other adverse outcomes and its relationship with mortality in disease has been explored in several studies (5). With liver cirrhosis, a clearer understanding of which HRV variables best predict mortality could lead to a better understanding of the disease and improved treatment outcomes.

HRV has been characterised by both linear tools and methods derived from non-linear dynamics. Linear methods such as the standard deviation of inter-beat intervals (SDNN) measure overall variability of the cardiac cycle, while spectral analysis decomposes fluctuations into different frequencies. Short-term versus long-term variability of the cardiac cycle can also be measured using geometrical methods such as the Poincare' plot $(6,7)$. Nonlinear tools have also been developed to analyse these fluctuations, such as regularity (sample entropy), complexity (multiscale entropy) and self-similarity (detrended fluctuation analysis) (8-10). These indices reflect different aspects of cardiac dynamics and there is no 
consensus as to which index performs best in the clinical setting (11). As for the relationship between HRV and survival, several HRV parameters have shown some promise. One such example is in congestive heart failure, where SDNN (an index of global HRV) identifies patients at higher risk of death due to progressive heart failure when the value is reduced (12). In patients with chronic kidney disease, a short-term HRV index was shown to be an independent risk factor for mortality (13). In patients with acute myocardial infarction shortterm fractal-like scaling exponent exhibited a better prognostic factor than a global HRV index (SDNN) (14). From these examples it is evident that there is benefit in looking at the relationship between HRV and mortality. However, different tools may be more or less suited to different disease conditions.

Looking at the relationship between HRV and survival in cirrhosis may be beneficial because total HRV is reduced in patients with cirrhosis (15). Additionally, an increase in severity of cirrhosis is associated with a reduction in HRV (15). Building on this, a few studies have looked at HRV as a physiomarker for predicting mortality in liver cirrhosis $(6,15,16)$. Mani et al. showed that the variable SD2 from the Poincaré analysis is significantly correlated with survival pre-transplantation, with the relative risk of death increasing by $7.7 \%$ with a $1 \mathrm{~ms}$ drop in this variable (6). Two studies looking at pre-transplantation data, also found a reduction in time-domain parameters when comparing survivors with non-survivors $(15,17)$. Chan et al., explored post-transplantation survival data to understand if HRV could better predict mortality 1 year after transplantation (16). Their findings showed a benefit in incorporating these parameters in their predictive tools (16). However, limited information remains available on which HRV parameter performs best in predicting mortality, and if they are independent of the severity of liver failure.

Several investigators have interpreted reduced HRV in cirrhosis as a marker for autonomic neuropathy. However, a physiological link between reduced HRV and autonomic dysfunction has not been demonstrated directly. This is important as a recent study by Monfredi et al has questioned the interpretation of reduced SDNN as an index of autonomic dysfunction (18). Monfredi et al. pointed out that there is a strong relationship between heart rate and SDNN, which does not depend on the autonomic nervous system (18). 
Monfredi et al. have also devised an HRV index known as corrected SDNN (cSDNN), which eliminates this HRV dependence (18).

The present study was designed to determine which of the aforementioned HRV physiomarkers derived from pre-transplant short-term heart rate recordings is best at predicting mortality in patients with cirrhosis. Additionally, if this ability to predict mortality is independent of the severity of hepatic failure.

\section{METHODS}

\subsection{Patients}

Ninety-eight patients with cirrhosis referred to the outpatient clinics of the Department of Medicine of the University of Padua from 29 June 2009 till 2 May 2011 were enrolled. Patients were excluded if they were under 16 or over 82 years of age; had cirrhosis on a transplanted liver, hepatocellular carcinoma, severe co-morbidity with short prognosis per se, a history of significant head injury, neurological or psychiatric disease other than hepatic encephalopathy, active alcohol misuse or acute infection. 74 patients (age \pm SD: $56 \pm 10.8$ years) met the inclusion/exclusion criteria. Patients were followed-up for 18 months and information was collected on the occurrence of death/liver transplantation. Patients who were transplanted for hepatic failure were considered 'non-survivor' on the day of transplantation (as they were in the immediate need of a new liver). Patients were followed-up for 18 months (mean \pm SD, $12.3 \pm 6.4$ months); if a patient was lost to follow up before the pre-defined time was up, the patient was censored on the date of latest available information. The flowchart of study procedures is shown in figure 1.

\subsection{Ethics statement}

The study protocol was approved by the Hospital of Padua Ethics Committee. All participants provided written, informed consent. This study was conducted according to the Declaration of Helsinki (Hong Kong Amendment) and Good Clinical Practice (European) guidelines. 


\subsection{Heart rate variability analysis}

A 10-min, lead II ECG was recorded at a sampling rate of $256 \mathrm{~Hz}$. The R peaks were detected, and the inter-beat interval time-series generated by using Chart software (Chart 5, AD-Instrument, Australia). The R-R interval series was visually inspected and 8-min, artefactfree continuous R-R interval sections were selected for analysis. All HRV indices were analysis using MATLAB (MathWorks) unless stated otherwise. The following indices were used for HRV analysis:

SDNN: Standard deviation of R-R intervals is calculated by the square root of the average of the squared individual differences. SDNN provides a measure of total HRV.

cSDNN: Corrected SDNN (cSDNN) for heart rate was therefore calculated using the following formula (18):

$$
\operatorname{cSDNN}=\frac{\text { SDNN }}{\mathrm{e}^{-\frac{\text { Heart rate }}{58.8}}}
$$

SD1: The Poincaré plot graphically represents the correlation between consecutive R-R intervals. We used the Poincaré plot to calculate short-term and long-term variability. The standard deviation of the points perpendicular to the line of identity (SD1) describes shortterm variability, which is mainly related to the effects of respiration on the vagal drive.

SD2: The standard deviation along the line of identity in the Poincare' plot (SD2) describes the long-term HRV variations. Many physiological factors including thermoregulation and the baroreflex loop contribute to long-term HRV.

Skewness: Increased asymmetry of the distribution of inter-beta interval around mean R-R interval has recently been reported in rats with cirrhosis and systemic inflammation (19). Skewness measures the asymmetry of the distribution of a time-series around its mean. The skewness for a symmetric distribution would be 0 , positive if the distribution has a longer tail to the right (i.e. more deceleration in cardiac rhythm than acceleration), and negative if 
the distribution has a longer tail to the left i.e. more acceleration in cardiac rhythm than deceleration).

Kurtosis: After mean, standard deviation and skewness, kurtosis is the fourth central moment of a distribution. It is a measure of whether the R-R interval data is heavy-tailed or light-tailed relative to a normal distribution when plotted as a histogram. In a similar way to skewness, kurtosis is a descriptor of the shape of distribution. Kurtosis represents how tall and sharp the distribution of data is. Data sets with low kurtosis have light tails. Data sets with high kurtosis tend to have heavy tails.

Spectral indices: Spectral analysis of the R-R interval time series was carried out by fast Fourier transformation. We employed Kubios software for spectral analysis without detrending (20). Three bands were identified: 1) a very low-frequency component (VLF: 0$0.04 \mathrm{~Hz}), 2$ ) a low-frequency component (LF: 0.04-0.15 Hz), and 3) a high-frequency component (HF: $0.15-0.4 \mathrm{~Hz}$ ). The LF/HF ratio was used as a measure of sympatho-vagal balance.

Sample entropy: The sample entropy (SampEn) estimates the degree of regularity of a timeseries such as inter-beat intervals. SampEn calculates the probability that an epoch of window length $\mathrm{m}$, with a degree of tolerance $r$, will be repeated at later time points. In the present study, $m=2$ and $r=0.2$ was used as recommended by Richman and Moorman (10).

Heart rate complexity (HRC): Complexity of a rhythm can be defined as "meaningful structural richness". Costa et al. extended the concept of entropy by incorporating multiple scales calculation of SampEn in a time-series (9). Such multiscale entropy method has been used to measure the complexity of different physiological time-series such as HRV and oxygen saturation variability (21). In present study, HRC was defined as the summation of entropies of scales from 1 to 20 as described (16).

Fractal-like exponents: Cardiac cycles exhibit scale-free or fractal-like dynamics which may be affected by systemic inflammation in cirrhosis (22). Detrended fluctuation analysis (DFA) is a classic method to quantify fractal-like correlation properties of physiological time-series 
(8). In this method, a linear relationship between $\log$ (fluctuations) and $\log$ (scale) indicates the presence of a fractal-like time series. The slope of this line $(\alpha)$ is the fractal-like exponent which can be separately calculated for short windows (scale $\leq 16$ ) and long windows (scale $>16$ ). Thus, we calculated two different values of $\alpha, \alpha 1$ and $\alpha 2$, which reflect short-term and long-term fractal-like exponents respectively (8).

\subsection{Measurement of inflammatory markers}

Plasma samples were collected from 37 patients with cirrhosis enrolled in the study and were stored at $-80^{\circ} \mathrm{C}$ until analysed for C-reactive protein (CRP), Tumour Necrosis- $\alpha$ (TNF- $\alpha$ ) and Interleukin-6 (IL-6) using routine laboratory protocols.

\subsection{Statistical analysis}

HRV indices of survivors and non-survivors were compared using either Student's t-test or the non-parametric Mann-Whitney U-test according to the distribution of the variable. Cox's proportional hazards regression was used for survival analysis. In this analysis, Cox's regression coefficient $(\beta)$ and the hazard ratio $\left(e^{\beta}\right)$ were calculated and the $p$-value for testing the null hypothesis $(\beta=0$, hazard ratio $=1)$ was determined using Wald test. Multivariate Cox's regression model was used to test if prediction of mortality by HRV indices is independent of the severity of hepatic failure.

The ROC curve was used to choose the best cut-off points for categorization of patients in the Kaplan-Meier graph based on their HRV indices. Log-rank (Mantel-Cox) test was used for survival analysis in the Kaplan-Meier graph. SPSS package (IBM) was used for statistical analysis. A p-value less than 0.05 is considered statistically significant. 


\section{RESULTS}

\subsection{Participants}

The data set used for this analysis was collected at the University of Padua and the patients were analysed in the 18-month follow-up. During the follow-up period, 24 patients (32\%) died or were transplanted for hepatic decompensation and were considered as "complete" cases. None of the recruited patients were transplanted for hepatocellular carcinoma.

The general characteristics of the study population are presented in Table 1 . There was no significant difference between the age and gender of survivors and non-survivors, however, both the MELD and Pugh scores were significantly different. When looking at the HRV characteristics, several parameters were significantly different when comparing the two groups (Table 2). The SDNN and cSDNN were significantly lower in non-survivors $(p<0.05)$ and as was the SD1 and SD2 ( $p<0.05)$. The spectral analysis variables VLF, VF, and HF were also lower in non-survivors $(p<0.05)$.

\subsection{HRV predictors of mortality}

The first part of the analysis was to determine which HRV parameters from Table 2 were linked with mortality and specifically, which one had the greatest predictive effect. From the variables listed in Table 3, it is evident that only $6 \mathrm{HRV}$ variables could predict mortality. As expected, both MELD score and Pugh's classification could also significantly predict mortality. Surprisingly, the traditional HRV indices (i.e. SDNN, SD1, SD2, VLF, HF), came up as significant as opposed to the novel complexity analysis tools. All HRV indices that were significant in the analysis were protective in nature, with an increase in variability being associated with a reduction in mortality. We also tested whether any of the HRV indices could distinguish between patients who died or underwent liver transplantation during the

follow up period. There was no statistically significant difference in any measured HRV index between these groups (Table S-1). In addition, Cox's regression analysis showed that none of HRV indices could predict death from liver transplantation during the follow up period (data not shown). 


\subsection{Analysis of the independence of HRV parameters in predicting mortality}

With the $6 \mathrm{HRV}$ variables that were significant in the first analysis, we combined the analysis with a measure of disease severity to determine if their ability to predict mortality was independent of severity of liver failure. Multivariate Cox's regression analysis showed that only two HRV indices were calculated to be independent of MELD: SD2 (Hazard ratio = $0.967, p<0.05$ ) and the cSDNN (Hazard ratio $=0.984, p<0.05$ ). The results of this analysis are presented in Table 4. This analysis was also repeated with the Pugh score and results were similar (Table 5). Among the HRV indices tested; SDNN, cSDNN and SD2 were all significant predictors of mortality independent of Pugh score.

We used death and liver transplantation as the main clinical end-points. MELD is often used to candidate patients for transplantation and is a clinical predictor of mortality in cirrhosis. We wondered whether HRV indices could influence the prognostic value of MELD score below or over 15 and the results showed that SD2 improved the prediction of survival in multivariate survival analysis with the decompensation index of MELD>15 (hazard ratio of SD2 was 0.961, $p=0.019)$.

\subsection{The effect of beta blocker administration on HRV parameters and survival}

Thirty-seven patients had received a beta adrenoceptor blocking agent for management of portal hypertension. We wondered if taking beta blockers would affect HRV indices or survival rates in patients with cirrhosis. Table S-2 demonstrates that basal heart rate was lower in cirrhotic patients who had received a beta receptor blocking agent $(60.7 \pm 1.6$ versus $68.7 \pm 1.7$ beats/min, $p<0.001$ ). However, none of the measured HRV indices were different between beta blocker-positive and beta blocker-negative groups. Besides, receiving beta blocker was not associated with an increase in mortality rate after 18 months follow up (hazard ratio $=1.917, p=0.144$, table 6).

\subsection{The effect of alcohol-related cirrhosis on HRV parameters and survival}


The aetiology of the cirrhosis was alcohol in 30 patients (40\%), chronic viral hepatitis (hepatitis B or C virus) in 28 patients (38\%), primary biliary cirrhosis in 1 patient (1.4\%), nonalcoholic steatohepatitis in 1 patient (1.4\%) and mixed in the remaining 14 patients (18.9\%). We wanted to determine if alcohol-related liver disease affected the HRV indices and mortality in patients with cirrhosis. Therefore, we compared the HRV indices between patients with alcohol-related cirrhosis and non-alcoholic cirrhosis. As shown in Table S-3, HRV indices were statistically identical in patients with alcohol-related cirrhosis compared with the rest of patients. In addition, having alcoholic-liver cirrhosis was not associated with an increase in mortality rate after 18 months follow up (Hazard ratio=0.924, $p=0.858$, table $6)$.

\subsection{Kaplan-Meier graph analysis}

Survival analysis on the three variables that showed clear independence from liver damage and showed a small predictive effect of mortality: SDNN, CSDNN, and SD2. A ROC analysis was conducted to find the cut-off value that provided the best possible trade off of sensitivity and specificity (data not shown). The cut off values for SDNN, cSDNN and SD2 were $20.4 \mathrm{~ms}, 56.7 \mathrm{~ms}$ and $25.5 \mathrm{~ms}$, respectively. This visualisation of the cut-off values is provided in the Kaplan-Meier plots for these three variables (Figures 2). In figure $2 \mathrm{~A}$, the cut off value for SDNN clearly separates into 2 groups which show marked difference in overall survival $(p<0.0001)$. The group below the cut-off have a significantly higher risk of mortality compared with the group above the cut-off. From figure $2 \mathrm{~B}$, it is evident to see a clear visual difference between the two groups that is statistically significant for cSDNN $(p<0.0001)$. The same is true in figure $2 \mathrm{C}$ for SD2, where survival prognosis was poorer for individuals below the cut-off value calculated $(p=0.0018)$.

\subsection{Inflammatory markers and HRV indices in patients with cirrhosis}

Previous reports have indicated that depressed HRV is associated with high plasma IL-6 levels in patients with systemic illnesses $(6,23,24)$. However, it is not known if elevated IL-6 levels explain the prognostic value of HRV in patients with cirrhosis. We firstly used Cox's regression analysis to see whether inflammatory markers can predict mortality in our 
patient's population. Our results indicated that among CRP, TNF- $\alpha$ and IL-6; only IL-6 could predict death in our study population (hazard ratio: 1.052, $p=0.003$, table 6). Further analysis showed that IL-6 level is independent of MELD in predicting mortality as both hazard ratios held significant in multivariate analysis (table 7). We also looked at the dependency of HRV indices to IL-6 in predicting mortality. The results showed that the prognostic value of HRV indices (cSDNN and SD2) depends on IL-6 level as only the hazard ratio of IL-6 held statistically significant in multivariate analysis along with HRV indices (table 7).

\section{DISCUSSION}

This study was aimed at determining which HRV variables best predict mortality in patients with cirrhosis. From the analysis carried out, the variables SDNN, cSDNN, and SD2 were found to be independent predictors of mortality in liver cirrhosis. The variables CSDNN and SD2 being the only variables independent from both markers of cirrhosis severity (i.e. MELD and Pugh scores). This result was surprising as these parameters are not the most recent methods developed to quantify heart rate complexity, especially when compared to DFA analysis or multiscale entropy analysis. Furthermore, they both show a protective role in reducing the likelihood of death in patients with cirrhosis.

The number of studies investigating this topic are limited but they all help build a better picture of the predictive ability of HRV analysis in cirrhosis $(6,15-17)$. Both Fleisher et al. and Ates et al. reported that a reduction in HRV could indicate a poor prognosis and mortality in cirrhosis $(15,17)$. Ates et al. showed that after a two year follow up period, HRV measurements were significantly lower in non-survivors compared to survivors (15). Our study expands this finding, as one of the measurements that was significantly different between the two groups was SDNN, which we confirmed through our analysis to be able to predict mortality. The predictive ability of long term HRV parameters (i.e. SD2) was known, as our team has previously reported that a 1-ms decrease in SD2 was associated with a 7.7\% increase in the relative risk of death in a group of cirrhotic patients referred to a tertiary liver unit in London (6). The current study confirms such findings in a larger and entirely 
separate cohort of patients, and also allowing for analysis of the relationship between this variable and the severity of hepatic failure. Chan et al. also looked at the relationship between the two in post-transplantation patients. Their findings showed that heart rate complexity and the deceleration capacity could accurately predict the mortality rate in patients 1-year post transplantation (16). However, they all have not looked whether this predictive ability of HRV is independent of cirrhosis severity, something novel in this study $(6,15,16)$. Chan et al. also showed that adding HRV tools to current methods of predicting mortality improves it, indicating that perhaps HRV is analysing an aspect additive to their predictor tool (16).

A second confounder that should be considered is the effect of mean heart rate on HRV. Although in this study age and heart rate were not significantly different between survivors and non-survivors, we know that patients with cirrhosis suffer from cardiac chronotropic incompetence (a component of cirrhotic cardiomyopathy) $(25,26)$. Thus, global HRV indices should be corrected for heart rate, in case this affects the results. cSDNN is a novel index which corrects for the effect of heart rate on SDNN (18). Our results on this were surprising as when we corrected SDNN for heart rate, the corrected value became independent of MELD. This suggests that either CSDNN or SD2 are the best physiomarkers for monitoring HRV in patients with cirrhosis.

We also considered beta blockers and aetiology of cirrhosis as possible confounder. Beta blockers can potentially alter heart rate dynamics and are routinely administer to patients with cirrhosis for management of portal hypertension. We wondered whether treatment with beta blocker had preferentially influenced a subgroup of patients with more advanced liver disease/portal pressure and hence unevenly affected HRV in patients with cirrhosis. However, our analysis showed that treatment with beta blocker only affects mean heart rate and does not have any significant effect either on HRV indices or survival. We also tested if alcohol-related cirrhosis affects heart rate dynamics differently in our study population. Likewise, alcohol-related liver disease was neither a predictor of mortality nor had any significant influence on HRV indices. Hence, this indicates that neither taking beta blocker or alcohol-related aetiology are confounders in our study. 
In order to physiologically interpret our findings, we need to understand what this independence means. The first hypothesis would be that the decrease in HRV which is associated with mortality is a sign of autonomic neuropathy. The LF variable represents sympathetic function while the HF variable is related to the effect of respiration on heart rate, which is mainly modulated by the vagus nerve. However, one would expect to see the best result with LF or HF, as they are clinically used to diagnose autonomic neuropathy in clinical settings such as diabetes (27). Surprisingly, LF, HF and the LF/HF ratio were all poor predictors of mortality, especially when compared to SD2. However, we cannot rule out the effect of autonomic neuropathy in cirrhosis simply from this finding.

One can consider other physiological components of long-term HRV. For example, the renin angiotensin system and thermoregulation and how they impact the VLF, another measure of long term HRV (28). Fleisher et al. showed that the VLF component is modulated by thermal stimuli related to core hypothermia and thermoregulatory activity (29). Additionally, as recent studies have showed thermoregulatory impairment in cirrhotic patients both in skin temperature recordings and core body temperature readings $(30,31)$. Perhaps the reduction in HRV independent of cirrhosis severity might be due to this impaired thermoregulation. However, as VLF was not affected perhaps this change could be due to another unknown factor. It appears that SD2 provides a better computational method for measurement of long-term HRV as VLF is heavily affected by de-trending R-R interval time-series in short-time recordings. The association between SD2 and core body temperature fluctuations in cirrhosis remains unstudied, something that can help explain these relationships.

An advantage of HRV analysis from short-time ECG recording (e.g. $8 \mathrm{~min}$ ) is that it can be incorporated into routine clinical monitors. Longer recording (e.g. 24 hours) are clinically less practical and would require heavy computational cost for automatic noise removal due to patient's movement or electrode detachment. The Task Force report suggests that 5-min inter-beat interval recording provides enough data for application of HRV in clinical practice and longer recording is not usually required (32). Calculation of both SD2 and cSDNN can be incorporated into routine clinical practice (e.g. in cardiac monitors). Chan et al. showed that incorporating HRV characteristics into a predictor tool with the MELD score, could predict 
post-transplant mortality in their patient population (16). It appears that reduced HRV does not necessarily respond positively to the liver transplant (33) and perhaps patients with reduced HRV would not benefit from liver transplantation. This suggests that reduced HRV could be considered as a comorbidity factor in the process of organ allocation (34). However, further study on post-transplantation survival is needed to better understand the role HRV plays with transplantation.

Although the mechanism of reduced HRV in cirrhosis is unclear, its correlation with IL-6 level suggests a mechanistic role for systemic inflammation (6). Cirrhosis shares mechanistic links with systemic inflammatory response syndrome and sepsis. In fact, reduced HRV is also a predictor of mortality in septic patients (35). Circulating IL-6 level is negatively correlated with HRV in both medical conditions (sepsis and cirrhosis) $(6,23)$. The results of the present study reveal that among inflammatory mediators, IL-6 is the only marker that could predict mortality in patients with cirrhosis. Similar to CSDNN and SD2, the predictive value of IL-6 is independent from MELD. Interestingly, the prognostic value of HRV indices (cSDNN and SD2) were influenced by IL-6 levels as shown in the multivariate Cox's regression analysis (table 7B). Hajiasgharzadeh et al. reported that infusion of IL-6 in mice decreases HRV and uncouples cardiac pacemaker from parasympathetic regulation (36). Based on these findings, increased IL-6 could explain reduced HRV in cirrhosis. However, the mechanistic link to survival is not clear. At least in theory, cirrhotic patients with reduced HRV might be more susceptible to develop severe sepsis. Such a hypothesis supports the role of the cholinergic anti-inflammatory pathway in prevention of organ failure in sepsis (37). Reduced HRV might mean lower activity in the neural anti-inflammatory pathway and hence higher chance of organ damage/failure after minor infection $(38,39)$. Although evidence for the contribution of this neural anti-inflammatory pathway in cirrhosis is emerging (40-42), the exact mechanistic link awaits further investigation. One limitation of our study is that the cause of death was not registered in our study. Future studies can look at the relationship between reduced HRV and cause of death in patients with cirrhosis.

Another limitation of our study is that we did not measure portal pressure, hyperdynamic circulation, echocardiogram or degree of cirrhotic cardiomyopathy in our patients. Cirrhosis is associated with peripheral vasodilatation that masks the latent cardiomyopathy at resting 
state (43). Thus, measurement of the degree of cardiomyopathy without intervention is not trivial in patients with cirrhosis. The relationship between reduced HRV and cirrhotic cardiomyopathy is not well understood. Studies in animal models of cirrhosis suggest that reduced HRV in rats with cirrhosis does not depend on impaired chronotropic responsiveness to beta-adrenergic (44) or cholinergic stimulation (22). Thus, the pathophysiologic link between reduced HRV and cirrhotic cardiomyopathy is missing. For this study we used HRV analysis that indicates the integrity of cardiovascular regulation. It makes sense to assume that the loss of integrity of cardiovascular control leads to mortality. Pioneering studies by Lee et al. demonstrate that central cardiovascular regulation is disordered in cirrhotic rats (45). Therefore, future studies could consider the relationship between reduced HRV and central cardiovascular regulatory mechanisms in the brain stem.

We conclude that both SD2 and corrected SDNN seem to be not only significant predictors of mortality in cirrhotic patients, but also to be predictors independent of liver damage and a useful tool for survival analysis.

\section{REFERENCES}

1. Mokdad AA, Lopez AD, Shahraz S, Lozano R, Mokdad AH, Stanaway J, et al. Liver cirrhosis mortality in 187 countries between 1980 and 2010: a systematic analysis. BMC Med. 2014 Dec;12(1):145.

2. Arvaniti V, D’Amico G, Fede G, Manousou P, Tsochatzis E, Pleguezuelo M, et al. Infections in Patients With Cirrhosis Increase Mortality Four-Fold and Should Be Used in Determining Prognosis. Gastroenterology. 2010 Oct;139(4):1246-1256.e5.

3. Zipprich A, Garcia-Tsao G, Rogowski S, Fleig WE, Seufferlein T, Dollinger MM. Prognostic indicators of survival in patients with compensated and decompensated cirrhosis. Liver Int. 2012 Oct;32(9):1407-14.

4. Montagnese S, De Rui M, Schiff S, Ceranto E, Valenti P, Angeli P, et al. Prognostic Benefit of the Addition of a Quantitative Index of Hepatic Encephalopathy to the MELD score: the MELD-EEG. Liver Int. 2015 Jan;35(1):58-64.

5. Tsuji H, Venditti FJ, Manders ES, Evans JC, Larson MG, Feldman CL, et al. Reduced Heart Rate Variability and Mortalit Risk in an Elderly Cohort The Framingham Heart 
Study. $1994 ; 878-83$.

6. Mani AR, Montagnese S, Jackson CD, Jenkins CW, Head IM, Stephens RC, et al.

Decreased heart rate variability in patients with cirrhosis relates to the presence and degree of hepatic encephalopathy. AJP Gastrointest Liver Physiol. 2009;296(2):G3308.

7. Hoshi RA, Pastre CM, Vanderlei LCM, Godoy MF. Poincaré plot indexes of heart rate variability: Relationships with other nonlinear variables. Auton Neurosci. 2013 Oct;177(2):271-4.

8. Peng C -K., Havlin S, Stanley HE, Goldberger AL. Quantification of scaling exponents and crossover phenomena in nonstationary heartbeat time series. Chaos An Interdiscip J Nonlinear Sci [Internet]. 1995 Mar [cited 2018 Jul 4];5(1):82-7. Available from: http://www.ncbi.nlm.nih.gov/pubmed/11538314

9. Costa $\mathrm{M}$, Costa $\mathrm{M}$, Goldberger $\mathrm{AL}$, Goldberger $\mathrm{AL}$, Peng $\mathrm{C}-\mathrm{K}$, Peng $\mathrm{C}-\mathrm{K}$, et al. Multiscale Entropy Analysis (MSE). Entropy. 2005;89(6):1-8.

10. Richman JS, Moorman JR. Physiological time-series analysis using approximate entropy and sample entropy. Am J Physiol Circ Physiol. 2000 Jun;278(6):H2039-49.

11. Sassi R, Cerutti S, Lombardi F, Malik M, Huikuri H V, Peng C-K, et al. Advances in heart rate variability signal analysis: joint position statement by the e-Cardiology ESC Working Group and the European Heart Rhythm Association co-endorsed by the Asia Pacific Heart Rhythm Society. Europace. 2015 Sep;17(9):1341-53.

12. Nolan J, Batin PD, Andrews R, Lindsay SJ, Brooksby P, Mullen M, et al. Prospective study of heart rate variability and mortality in chronic heart failure: results of the United Kingdom heart failure evaluation and assessment of risk trial (UK-heart). Circulation. 1998 Oct;98(15):1510-6.

13. Drawz PE, Babineau DC, Brecklin C, He J, Kallem RR, Soliman EZ, et al. Heart Rate Variability Is a Predictor of Mortality in Chronic Kidney Disease: A Report from the CRIC Study. Am J Nephrol. 2013;38(6):517-28.

14. Mäkikallio TH, Høiber S, Køber L, Torp-Pedersen C, Peng CK, Goldberger AL, et al. Fractal analysis of heart rate dynamics as a predictor of mortality in patients with depressed left ventricular function after acute myocardial infarction. TRACE Investigators. TRAndolapril Cardiac Evaluation. Am J Cardiol. 1999 Mar;83(6):836-9.

15. Ates F, Topal E, Kosar F, Karincaoglu M, Yildirim B, Aksoy Y, et al. The relationship of 
heart rate variability with severity and prognosis of cirrhosis. Dig Dis Sci. 2006;51(9):1614-8.

16. Chan K-C, Yeh J-R, Sun W-Z. The role of autonomic dysfunction in predicting 1-year mortality after liver transplantation. Liver Int. 2017;37(8):1239-48.

17. Fleisher LA, Fleckenstein JF, Frank SM, Thuluvath PJ. Heart rate variability as a predictor of autonomic dysfunction in patients awaiting liver transplantation. Dig Dis Sci. 2000 Feb;45(2):340-4.

18. Monfredi O, Lyashkov AE, Johnsen A-B, Inada S, Schneider H, Wang R, et al. Biophysical Characterization of the Underappreciated and Important Relationship Between Heart Rate Variability and Heart RateNovelty and Significance. Hypertension [Internet]. 2014;64(6):1334-43. Available from: http://hyper.ahajournals.org/lookup/doi/10.1161/HYPERTENSIONAHA.114.03782

19. Taghipour M, Eftekhari G, Haddadian Z, Mazloom R, Mani M, Mani AR. Increased sample asymmetry and memory of cardiac time-series following endotoxin administration in cirrhotic rats. Physiol Meas. 2016 Nov;37(11):N96-104.

20. Tarvainen MP, Niskanen J-P, Lipponen JA, Ranta-aho PO, Karjalainen PA. Kubios HRV Heart rate variability analysis software. Comput Methods Programs Biomed. 2014 Jan;113(1):210-20.

21. Bhogal AS, Mani AR. Pattern Analysis of Oxygen Saturation Variability in Healthy Individuals: Entropy of Pulse Oximetry Signals Carries Information about Mean Oxygen Saturation. Front Physiol. 2017 Aug;8:555.

22. Haddadian Z, Eftekhari G, Mazloom R, Jazaeri F, Dehpour AR, Mani AR. Effect of endotoxin on heart rate dynamics in rats with cirrhosis. Auton Neurosci Basic Clin. 2013;177(2):104-13.

23. Tateishi Y, Oda S, Nakamura M, Watanabe K, Kuwaki T, Moriguchi T, et al. Depressed heart rate variability is associated with high IL-6 blood level and decline in the blood pressure in septic patients. Shock [Internet]. 2007 [cited 2018 Aug 24];28(5):549-53. Available from: https://insights.ovid.com/pubmed?pmid=18075483

24. González-Clemente J-M, Vilardell C, Broch M, Megia A, Caixàs A, Giménez-Palop O, et al. Lower heart rate variability is associated with higher plasma concentrations of IL-6 in type 1 diabetes. Eur J Endocrinol [Internet]. 2007 Jul 1 [cited 2018 Aug 24];157(1):31-8. Available from: http://www.ncbi.nlm.nih.gov/pubmed/17609399 
25. Wiese S, Hove JD, Bendtsen F, Møller S. Cirrhotic cardiomyopathy: pathogenesis and clinical relevance. Nat Rev Gastroenterol Hepatol. 2014 Mar;11(3):177-86.

26. Møller S, Lee SS. Cirrhotic cardiomyopathy. J Hepatol. 2018 Apr;0(0).

27. Chessa M, Butera G, Lanza GA, Bossone E, Delogu A, De Rosa G, et al. Role of Heart Rate Variability in the Early Diagnosis of Diabetic Autonomic Neuropathy in Children. Herz. 2002 Dec;27(8):785-90.

28. Akselrod S, Gordon D, Ubel FA, Shannon DC, Berger AC, Cohen RJ. Power spectrum analysis of heart rate fluctuation: a quantitative probe of beat-to-beat cardiovascular control. Science. $1981 \mathrm{Jul} ; 213(4504): 220-2$.

29. Fleisher L a, Frank SM, Sessler DI, Cheng C, Matsukawa T, Vannier C a. Thermoregulation and heart rate variability. Clin Sci (Lond). 1996;90(2):97-103.

30. Garrido M, Saccardo D, De Rui M, Vettore E, Verardo A, Carraro P, et al. Abnormalities in the 24-hour rhythm of skin temperature in cirrhosis: Sleep-wake and general clinical implications. Liver Int. 2017;37(12):1833-42.

31. Mani AR, Mazloom R, Haddadian Z, Montagnese S. Body temperature fluctuation analysis in cirrhosis. Liver Int. 2018;38(2):378-9.

32. Task Force of The European Society of Cardiology and The North American, Society of Pacing and Electrophysiology (Membership. Guidelines Heart rate variability Standards of measurement, physiological interpretation, and clinical use. Eur Heart J [Internet]. 1996 [cited 2018 Aug 25];17:354-81. Available from: https://www.escardio.org/static_file/Escardio/Guidelines/ScientificStatements/guidelines-Heart-Rate-Variability-FT-1996.pdf

33. Baratta L, Tubani L, Merli M, Labbadia F, Facchini D, De Marco R, et al. Long-term effect of liver transplantation on cirrhotic autonomic cardiac dysfunction. Dig Liver Dis. 2010;42(2):131-6.

34. Bhogal AS, Montagnese S, Mani AR. The consideration of heart rate complexity as a co-morbidity factor for liver transplantation selection procedures. Liver Int. 2018 Feb;38(2):380-380.

35. De Castilho FM, Luiz A, Ribeiro P, Luiz J, Da Silva P, Nobre V, et al. Heart rate variability as predictor of mortality in sepsis: A prospective cohort study. 2017 [cited 2018 Aug 13]; Available from: https://doi.org/10.1371/journal.pone.0180060

36. Hajiasgharzadeh K, Mirnajafi-Zadeh J, Mani AR. Interleukin-6 impairs chronotropic 
responsiveness to cholinergic stimulation and decreases heart rate variability in mice. Eur J Pharmacol. 2011;673(1-3).

37. Olofsson PS, Rosas-Ballina M, Levine YA, Tracey KJ. Rethinking inflammation: Neural circuits in the regulation of immunity. Immunol Rev [Internet]. 2012 [cited 2018 Aug 13];248(1):188-204. Available from: https://www.ncbi.nlm.nih.gov/pmc/articles/PMC4536565/pdf/nihms488462.pdf

38. Mazloom R, Eftekhari G, Rahimi M, Khori V, Hajizadeh S, Dehpour AR, et al. The role of $\alpha 7$ nicotinic acetylcholine receptor in modulation of heart rate dynamics in endotoxemic rats. PLoS One. 2013;8(12).

39. Eftekhari G, Ahmadi Soleimani SM, Mani AR. Heart rate variability, vagal activity and the pulse of inflammation. J Intern Med. 2013;274(5).

40. Eftekhari G, Hajiasgharzadeh K, Ahmadi-Soleimani SM, Dehpour AR, Semnanian S, Mani AR. Activation of central muscarinic receptor type 1 prevents development of endotoxin tolerance in rat liver. Eur J Pharmacol. 2014;740.

41. Hajiasgharzadeh K, Tavangar SM, Javan M, Dehpour AR, Mani AR. Does hepatic vagus nerve modulate the progression of biliary fibrosis in rats? Auton Neurosci Basic Clin. 2014;185.

42. Hajiasgharzadeh K, Baradaran B. Cholinergic anti-inflammatory pathway and the liver [Internet]. Vol. 7, Advanced Pharmaceutical Bulletin. 2017 [cited 2018 Jul 23]. p. 50713. Available from: http://apb.tbzmed.ac.ir

43. Liu H, Jayakumar S, Traboulsi M, Lee SS. Cirrhotic cardiomyopathy: Implications for liver transplantation. Liver Transplant. 2017 Jun;23(6):826-35.

44. Mani AR, Ippolito S, Ollosson R, Moore KP. Nitration of cardiac proteins is associated with abnormal cardiac chronotropic responses in rats with biliary cirrhosis. Hepatology. 2006 Apr;43(4):847-56.

45. Song D, Sharkey KA, Breitman DR, Zhang Y, Lee SS. Disordered central cardiovascular regulation in portal hypertensive and cirrhotic rats. Am J Physiol Liver Physiol. 2001 Mar;280(3):G420-30. 


\section{TABLES}

Table 1. The mean characteristics for the study population.

\section{Survivors Non-Survivors p-value}

\begin{tabular}{lccc}
\hline Number & 50 & 24 & - \\
Gender $(\delta / q)$ & $65.3 \%$ & $68.2 \%$ & 0.999 \\
Age & $56.6 \pm 1.6$ & $56.3 \pm 2.2$ & 0.888 \\
MELD a & $12.6 \pm 0.7$ & $17.6 \pm 1.4$ & $\mathbf{0 . 0 0 2}$ \\
Pugh & $7.6 \pm 0.3$ & $9.8 \pm 0.5$ & $<\mathbf{0 . 0 0 1}$ \\
\hline
\end{tabular}

The data are expressed as \pm SEM. Level of significance set at $p<0.05$. Fisher's exact test was used to compere the gender. ${ }^{a}$ Mann-Whitney-U test used due positive results with Levene's test of equal variances.

Table 2. The mean HRV characteristics for the study population.

\begin{tabular}{lccc} 
& Survivors & Non-Survivors & p-value \\
\hline Number & 50 & 24 & - \\
Mean Heart rate (bpm) & $62.6 \pm 1.6$ & $67.5 \pm 1.9$ & 0.064 \\
SDNN (ms)* & $29.1 \pm 2.1$ & $18.9 \pm 2.0$ & $\mathbf{0 . 0 0 2}$ \\
cSDNN (ms) & $81.9 \pm 5.0$ & $68.1 \pm 5.4$ & $\mathbf{0 . 0 0 5}$ \\
SD1 (ms)* & $15.1 \pm 1.4$ & $9.5 \pm 1.3$ & $\mathbf{0 . 0 0 6}$ \\
SD2 (ms) & $37.7 \pm 2.7$ & $24.8 \pm 2.5$ & $\mathbf{0 . 0 0 3}$ \\
Kurtosis & $5.1 \pm 0.8$ & $4.1 \pm 0.4$ & 0.395 \\
Skewness & $-0.4 \pm 0.07$ & $-0.3 \pm 0.09$ & 0.194 \\
VLF (ms $\left.{ }^{2}\right)^{*}$ & $483 \pm 73$ & $205 \pm 38$ & $\mathbf{0 . 0 1 4}$ \\
LF (ms ${ }^{*}$ & $212 \pm 44$ & $89 \pm 33$ & $\mathbf{0 . 0 1 1}$ \\
HF (ms $\left.{ }^{2}\right)^{*}$ & $239 \pm 46$ & $80 \pm 30$ & $\mathbf{0 . 0 0 2}$ \\
LF/HF & $0.42 \pm 0.22$ & $1.52 \pm 0.21$ & 0.777 \\
SampEn & $1.59 \pm 0.06$ & $1.62 \pm 0.10$ & 0.581 \\
HRC & $46.1 \pm 1.9$ & $49.6 \pm 2.6$ & 0.306 \\
$\boldsymbol{\alpha 1}$ & $0.96 \pm 0.04$ & $1.01 \pm 0.04$ & 0.388 \\
$\boldsymbol{\alpha 2}$ & $0.99 \pm 0.03$ & $1.03 \pm 0.03$ & 0.388 \\
\hline
\end{tabular}

The data are expressed as mean \pm SEM. CSDNN is the corrected SDNN for heart rate using Monfredi et al. formula. HRC is the heart rate complexity calculated using integration of multiscale entropy analysis. $\alpha 1$ and $\alpha 2$ are the short-term and long-term fractal-like scaling exponent respectively calculated using detrended fluctuation analysis (DFA). Level of significance set at $p<0.05$. * Mann-Whitney-U test used due positive results with Levene's test of equal variances. 
Table 3. The predictive effect of HRV parameters on mortality

\begin{tabular}{lccccc} 
& $\boldsymbol{\beta}$ & SEM & Hazard ratio & Cl & p-value \\
\hline Age & & & & & \\
MELD & 0.004 & 0.019 & 1.004 & $0.967-1.042$ & $<.828$ \\
Pugh & 0.165 & 0.037 & 1.179 & $1.097-1.268$ & $<.001$ \\
Mean Heart rate & 0.478 & 0.1 & 1.612 & $1.324-1.963$ & $<0.001$ \\
SDNN & 0.029 & 0.019 & 1.029 & $0.993-1.067$ & 0.119 \\
CSDNN & -0.067 & 0.022 & 0.935 & $0.895-0.977$ & $\mathbf{0 . 0 0 3}$ \\
SD1 & -0.025 & 0.008 & 0.975 & $0.959-0.991$ & $\mathbf{0 . 0 0 2}$ \\
SD2 & -0.085 & 0.033 & 0.919 & $0.861-0.980$ & $\mathbf{0 . 0 1 0}$ \\
Kurtosis & -0.052 & 0.017 & 0.95 & $0.918-0.982$ & $\mathbf{0 . 0 0 3}$ \\
Skewness & -0.103 & 0.117 & 0.902 & $0.810-1.112$ & 0.381 \\
VLF & 0.69 & 0.432 & 1.994 & $0.710-3.824$ & 0.110 \\
LF & -0.003 & 0.001 & 0.997 & $0.995-0.999$ & $\mathbf{0 . 0 2 0}$ \\
HF & -0.003 & 0.002 & 0.997 & $0.993-1.000$ & 0.071 \\
LF/HF & -0.005 & 0.002 & 0.995 & $0.991-0.999$ & $\mathbf{0 . 0 2 3}$ \\
SampEn & 0.049 & 0.131 & 1.05 & $0.912-1.357$ & 0.710 \\
HRC & 0.458 & 0.512 & 1.581 & $0.467-3.257$ & 0.371 \\
$\boldsymbol{\alpha 1}$ & 0.015 & 0.014 & 1.015 & $0.987-1.044$ & 0.294 \\
$\boldsymbol{\alpha 2}$ & 0.645 & 0.797 & 1.906 & $0.400-9.090$ & 0.418 \\
\hline & 1.095 & 2.376 & $0.278-20.30$ & 0.429 \\
\hline & 0.866 & & & \\
\hline
\end{tabular}

$\beta$ is the coefficient of Cox's regression analysis. SEM is the standard error of the mean of $\beta$, Hazard ratio $=\operatorname{Exp}(\beta)=e^{\beta} . \mathrm{Cl}=95 \%$ confidence interval of the hazard ratio.

Table 4. The independence of the HRV parameters from the MELD score in predicting mortality. 


\begin{tabular}{lccccc} 
& $\boldsymbol{\beta}$ & SEM & Hazard ratio & Cl & p-value \\
\hline SDNN & -0.042 & 0.022 & 0.959 & $0.919-1.000$ & 0.051 \\
CSDNN & -0.017 & 0.008 & 0.984 & $0.968-0.999$ & $\mathbf{0 . 0 4 8}$ \\
SD1 & -0.045 & 0.032 & 0.956 & $0.897-1.018$ & 0.162 \\
SD2 & -0.034 & 0.017 & 0.967 & $0.935-0.999$ & $\mathbf{0 . 0 4 3}$ \\
HF & -0.003 & 0.002 & 0.997 & $0.995-1.001$ & 0.137 \\
VLF & -0.002 & 0.001 & 0.998 & $0.996-1.000$ & 0.075 \\
\hline
\end{tabular}

MELD score was significant in the analysis when compared with the other variables listed, hence the validity of the data. $\mathrm{Cl}=95 \%$ confidence interval of the hazard ratio.

Table 5. The independence of the HRV parameters from the Pugh Classification in predicting mortality.

\begin{tabular}{lccccc} 
& $\boldsymbol{\beta}$ & SEM & Hazard ratio & $\mathbf{C l}$ & $\boldsymbol{p}$-value \\
\hline SDNN & -0.042 & 0.02 & 0.958 & $0.922-0.997$ & $\mathbf{0 . 0 3 3}$ \\
cSDNN & -0.018 & 0.008 & 0.982 & $0.967-0.998$ & $\mathbf{0 . 0 2 4}$ \\
SD1 & -0.054 & 0.028 & 0.948 & $0.896-1.002$ & 0.059 \\
SD2 & -0.034 & 0.016 & 0.967 & $0.937-0.997$ & $\mathbf{0 . 0 3 4}$ \\
HF & -0.003 & 0.002 & 0.997 & $0.993-1.000$ & 0.053 \\
VLF & -0.002 & 0.001 & 0.998 & $0.996-1.000$ & 0.131 \\
\hline
\end{tabular}

Pugh score was significant in the analysis when compared with the other variables listed, hence the validity of the data. $\mathrm{Cl}=95 \%$ confidence interval of the hazard ratio. 
Table 6. The predictive effect of the aetiology of cirrhosis, receiving beta blocker and plasma markers of inflammation on mortality

\begin{tabular}{lccccc} 
& $\boldsymbol{\beta}$ & SEM & Hazard ratio & Cl & p-value \\
\hline Aetiology (alcoholic & & & & & \\
versus non-alcoholic) & -0.080 & 0.444 & 0.924 & $0.387-2.203$ & 0.858 \\
Beta blocker & 0.651 & 0.445 & 1.917 & $0.802-4.583$ & 0.144 \\
C-Reactive Protein (CRP) & 0.001 & 0.012 & 1.001 & $0.978-1.250$ & 0.911 \\
TNF- $\boldsymbol{\alpha}$ & 0.021 & 0.033 & 1.021 & $0.957-1.090$ & 0.527 \\
IL-6 & 0.051 & 0.017 & 1.052 & $1.017-1.88$ & $\mathbf{0 . 0 0 3}$
\end{tabular}

$\beta$ is the coefficient of Cox's regression analysis. SEM is the standard error of the mean of $\beta$, Hazard ratio $=\operatorname{Exp}(\beta)=e^{\beta}, \mathrm{Cl}=95 \%$ confidence interval of the hazard ratio.

Table 7. Multivariate survival analysis to test the independence of MELD (A) and HRV indices (B) from plasma IL-6 levels in predicting mortality.

\begin{tabular}{|c|c|c|c|c|c|}
\hline A & $\boldsymbol{\beta}$ & SEM & Hazard ratio & $\mathrm{Cl}$ & p-value \\
\hline MELD & 0.115 & 0.048 & 1.122 & \multirow{3}{*}{$\begin{array}{l}1.021-1.231 \\
1.002-1.077\end{array}$} & 0.016 \\
\hline IL-6 & 0.038 & 0.018 & 1.039 & & 0.038 \\
\hline \multicolumn{5}{|l|}{ B } & \\
\hline cSDNN & -0.015 & 0.011 & 0.985 & $0.964-1.006$ & 0.152 \\
\hline IL-6 & 0.054 & 0.018 & 1.056 & $1.018-1.094$ & 0.003 \\
\hline SD2 & -0.030 & 0.022 & 0.971 & $0.929-1.014$ & 0.176 \\
\hline IL-6 & 0.054 & 0.019 & 1.056 & $1.016-1.095$ & 0.004 \\
\hline
\end{tabular}

$\beta$ is the coefficient of Cox's regression analysis. SEM is the standard error of the mean of $\beta$, Hazard ratio $=\operatorname{Exp}(\beta)=e^{\beta}, \mathrm{Cl}=95 \%$ confidence interval of the hazard ratio. 


\section{FIGURE LEGEND:}

Figure 1. The flowchart of study procedures.

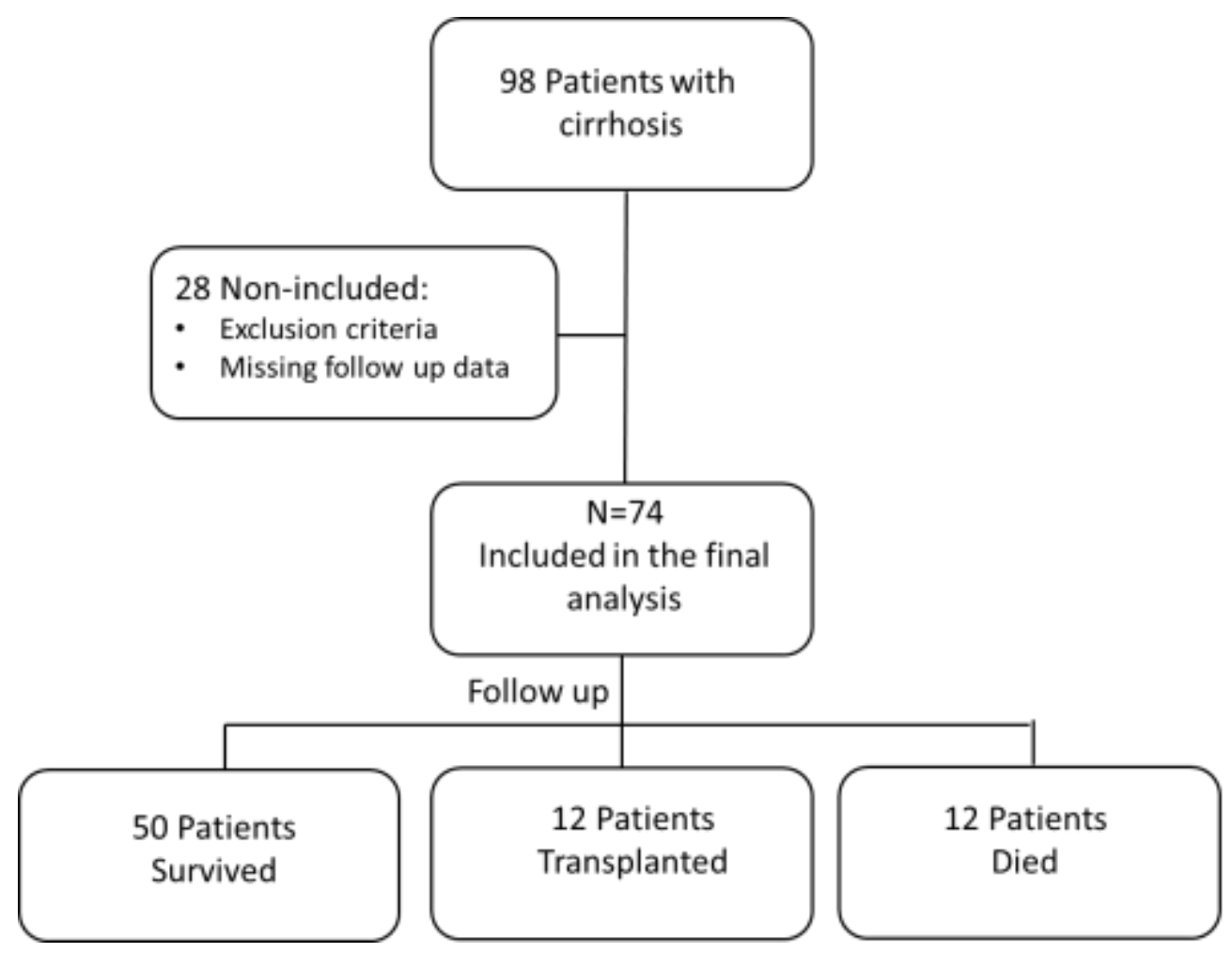


Figure 2. Survival graphs depicting the overall survival for patients with cirrhosis above and below SDNN, CSDNN or SD2 cut off values. A. SDNN (Log-rank test, Chi square $=15.06$, p $<0.0001)$. B. cSDNN is the corrected SDNN for heart rate using Monfredi et al., (Log-rank test, Chi square $=18.96, p<0.0001)$. C. SD2 indicates long-term HRV and is calculated using Poincare' plot (Log-rank test, Chi square $=9.74, p=0.0018)$.
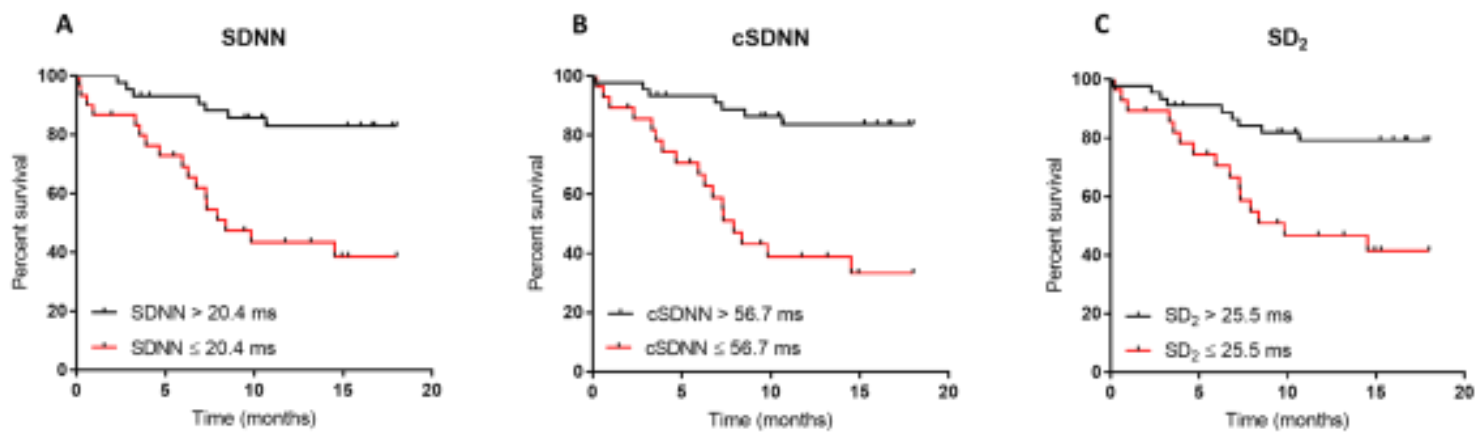

\begin{tabular}{|l|c|c|c|c|c|c|c|}
\hline Number of subjects at riak \\
\hline Time (montis) & 0 & 3 & 6 & 9 & 12 & 15 & 18 \\
\hline SONW $>20.4 \mathrm{~ms}$ & 43 & 42 & 30 & 30 & 30 & 30 & 24 \\
\hline SONN $20.4 \mathrm{~ms}$ & 31 & 27 & 21 & 15 & 11 & 8 & 6 \\
\hline
\end{tabular}

\begin{tabular}{|c|c|c|c|c|c|c|c|c|c|c|c|c|c|c|c|}
\hline \multicolumn{8}{|c|}{ Number of suejects of rhak } & \multicolumn{8}{|c|}{ Number of suthjocts at nak } \\
\hline Time [montis] & 0 & 3 & 6 & 9 & 12 & 15 & 19 & Tme inenetsis) & 0 & 3 & 6 & 9 & 12 & 15 & 18 \\
\hline esDNN > $56.7 \mathrm{~ms}$ & 45 & 44 & 41 & $\$$ & $\$ 2$ & 32 & 25 & $5 \mathrm{D}_{2}>25.5 \mathrm{~ms}$ & 45 & 43 & $\infty$ & $\boldsymbol{x}$ & 30 & 30 & 24 \\
\hline CSDNN $56.7 \mathrm{~ms}$ & 29 & 25 & 19 & 13 & 9 & 6 & 5 & $80,525.5 \mathrm{~ms}$ & 29 & 26 & 20 & 16 & 11 & 8 & 6 \\
\hline
\end{tabular}

